\title{
A NONLOCAL MODEL OF CHIRAL DYNAMICS
}

\author{
B. HOLDOM, J. TERNING and K. VERBEEK \\ Department of Physics, University of Toronto, Toronto, Ontario, Canada M5S $1 A 7$
}

Received 14 August 1989; revised manuscript received 10 October 1989

\begin{abstract}
We consider a nonlocal generalization of the nonlinear $\sigma$ model. Our chirally symmetric model couples quarks with self-energy $\Sigma(p)$ to Goldstone bosons (GBs). By integrating out the quarks we obtain a chiral lagrangian, the parameters of which are finite integrals of $\Sigma(p)$. We find that chiral symmetry is not sufficient to derive the well-known Pagels-Stokar formula for the GB decay constant. We reproduce the Wess-Zumino term and we illustrate the dependence of other four derivative coefficients on $\Sigma(p)$.
\end{abstract}

We wish to consider a simple model of quarks with a nonlocal and nonlinear coupling to a Goldstone boson (GB) field. We hope to capture more of the physics of chiral symmetry breaking in gauge theories than does a standard nonlinear sigma model with the following GB-quark coupling:

$m \bar{\psi}(x) V(x) \psi(x), \quad V(x) \equiv \exp \left[-2 \mathrm{i} \pi(x) \gamma_{5} / f_{\pi}\right]$.

Our model consists of $N$ flavors of quarks each with $N_{c}$ "colors" interacting with a GB field via a bilocal coupling:

$\mathscr{L}_{\psi}(x, y)=\bar{\psi}(x) \delta(x-y) \not \psi \psi(y)+\bar{\psi}(x) \Sigma_{\pi}(x, y) \psi(y)$.

$\Sigma_{\pi}$ is a nonlinear function, to be determined, of the GB field $\pi(x) \equiv \sum_{a} \lambda_{a} \pi_{a}(x)$ [ $\lambda_{a}$ are generators of SU $(N)$, $\left.\operatorname{Tr} \lambda_{a} \lambda_{b}=\frac{1}{2} \delta_{a b}\right] . \Sigma_{\pi}$ is a nontrivial matrix only in the $\operatorname{SU}(N)_{\mathrm{L}} \times \mathrm{SU}(N)_{\mathrm{R}}$ flavor space. We shall require that $\pi(x)$ transforms under chiral transformations in the standard nonlinear manner, and that $\Sigma_{\pi}$ transforms in such a way that $\mathrm{SU}(N)_{\mathrm{L}} \times \mathrm{SU}(N)_{\mathrm{R}}$ is a global symmetry. The model also has a vector $\mathrm{U}\left(N_{\mathrm{c}}\right)$ "color" symmetry, but we leave this ungauged. We will work in euclidean space.

We are considering a bilocal coupling since for vanishing $\pi$ we shall require that $\Sigma_{\pi}(x, y) \Rightarrow \Sigma(x-y) \delta_{i j}(i, j=1$, $\ldots, N) . \Sigma(x-y)$ is the Fourier transform of the quark self-energy $\Sigma(p)$, a function of $p^{2}$. In a gauge theory with zero bare mass quarks the dynamical self-energy $\Sigma(p)$ is the order parameter of chiral symmetry breaking. The appearance of $\Sigma(x-y)$ in our model allows us to input a realistic self-energy as would be determined for example by some approximation to the gap equation in a gauge theory. In contrast, a local sigma model with fermions has $\Sigma(x-y) \propto \delta(x-y)$ and $\Sigma(p)=$ constant; this is not representative of a dynamical mass in a gauge theory. Since we use $\Sigma(x-y)$ to represent an order parameter, it is then natural that the Goldstone fields appear as fluctuations in the orientation of $\Sigma(x-y)$ in $\mathrm{SU}(N)_{\mathrm{L}} \times \mathrm{SU}(N)_{\mathrm{R}}$.

The statement that GBs should appear as "fluctuations of the order parameter" will define our model. It is equivalent to insisting that $\bar{\psi}(x) \Sigma_{\pi}(x, y) \psi(y)$ should reduce to (1) in the special case that $\Sigma(x-y)=m \delta(x-y)$. This is a nontrivial restriction since various derivative couplings are also allowed in a local nonlinear sigma model; we intentionally omit the nonlocal generalizations of all such terms.

The GB kinetic terms and self-couplings will be generated dynamically. In fact we will integrate out the quark fields and show that a derivative expansion yields a local chiral lagrangian for the GBs:

$\exp \left(-\int \mathrm{d}^{4} x \mathscr{L}_{\text {eff }}(\pi(x))\right) \equiv \int \mathscr{D} \bar{\psi} \mathscr{D} \psi \exp \left(-\int \mathrm{d}^{4} x \mathrm{~d}^{4} y \mathscr{L}_{\psi}(x, y)\right)$ 
$\int \mathrm{d}^{4} x \mathscr{L}_{\mathrm{eff}}(\pi(x))=-\operatorname{tr} \log S_{\pi}^{-1}$,

$S_{\pi}^{-1}(x, y) \equiv \oint_{x} \delta(x-y)+\Sigma_{\pi}(x, y)$.

We will express the coefficients of the various terms in the chiral lagrangian as finite integrals involving $\Sigma(p)$. In particular we obtain an expression for $f_{\pi}$, the GB decay constant, and we reproduce the known coefficient of the Wess-Zumino term.

We also consider other four derivative terms of interest for pion-pion scattering and skyrmion solutions. Other authors have obtained the Wess-Zumino term and other four derivative terms in various one fermionloop calculations in local models [1]. But these models are unable to address the two derivative terms, ie. the calculation of $f_{\pi}$. Other nonlocal precursors of chiral lagrangians have also been considered [2].

Different gauge theories with various particle contents will yield differing $\Sigma(p)$. For example walking technicolor theories are constructed to yield a $\Sigma(p)$ which falls more slowly than in QCD. Our model may be useful in indicating how physical low energy quantities depend on the behavior of $\Sigma(p)$.

We must first determine $\Sigma_{\pi}(x, y)$. (1) implies that a global $\mathrm{SU}(N)_{\mathrm{L}} \times \mathrm{SU}(N)_{\mathrm{R}}$ rotation of $V(x)$ gives the standard nonlinear transformation law for $\pi(x)$. We will thus construct $\Sigma_{\pi}(x, y)$ from $V(x)$ and $V(y)$ so as to ensure global chiral invariance. Also, hermiticity requires that $\Sigma_{\pi}(x, y)=\Sigma_{\pi}(y, x)$.

We will consider the following form for $\Sigma_{\pi}(x, y)$ :

$\Sigma_{\pi}(x, y)=\Sigma(x-y) T \cdot \frac{1}{2}\left\{\left[a V(x)+b V(x) V(y)^{\dagger} V(x)+c V(x) V(y)^{\dagger} V(x) V(y)^{\dagger} V(x)+\ldots\right]+[x \leftrightarrow y]\right\}$.

$T$ is some function, symmetric in $x$ and $y$, of traces of strings of $\ldots V(x) V(y)^{\dagger} \ldots$ or ...V(y)V(x) ${ }^{\dagger} \ldots$ such that $T(V=1)=1$. The constants satisfy

$a+b+c+\ldots=1$.

The single GB terms in $\Sigma_{\pi}(x, y)$ are completely specified in (6):

$\Sigma_{\pi}(x, y)=\Sigma(x-y)\left\{1-\mathrm{i} \gamma_{5}[\pi(x)+\pi(y)] / f_{\pi}+(\right.$ higher order in $\left.\pi)\right\}$.

The higher order terms in (8) are constrained but are not completely determined. This arbitrariness in the multi-GB couplings will have implications below. But ( 8 ) does determine the vertex function for a single GB of momentum $q$ coupling to a quark and antiquark:

$G_{\pi a}(p, p+q)=\mathrm{i} \lambda_{a} \gamma_{5}[\Sigma(p+q)+\Sigma(p)] / f_{\pi}$.

We may relate this result to chiral symmetry breaking in a gauge theory by recalling the WT identity for the axial current vertex function $\Gamma_{5 a}^{\mu}$ :

$-\mathrm{i} q_{\mu} \Gamma_{5 a}^{\mu}(p, p+q)=S_{\mathrm{f}}^{-1}(p+q) \lambda_{a} \gamma_{5}+\lambda_{a} \gamma_{5} S_{\mathrm{f}}^{-1}(p)$.

$S_{\mathrm{f}}(p)$ is the full quark propagator, $S_{\mathrm{f}}(p) \equiv\{Z(p)[-\mathrm{i} \not p+\Sigma(p)]\}^{-1}$. The presence of $\Sigma(p)$ signal chiral symmetry breaking; it implies that $\Gamma_{5 a}^{\mu}$ contains a term with a pole in $q^{2}$. This pole may be identified with a GB with axial current coupling $\left\langle 0\left|J_{5 a}^{\mu}\right| \pi_{b}(q)\right\rangle \equiv-q^{\mu} f_{\pi} \delta_{a b}$. Then (10) constrains the GB coupling to quarks. In the approximation where $Z(p)=1$ and where the nonpole part of $\Gamma_{5 a}^{\mu}$ is $\tilde{\Gamma}_{5 a}^{\mu}(p, p+q)=\lambda_{a} \gamma^{\mu} \gamma_{5}$ then (10) implies the same $\mathrm{GB}$ vertex function as in (9).

This approximation coincides with the "dynamical perturbation theory" of Pagels and Stokar [3] In lowest order all amplitudes which do not vanish in perturbation theory (in the gauge coupling) are set to their free field values. Amplitudes with vanish in perturbation theory but which nevertheless arise dynamically, such as $\Sigma(p)$, are retained. The result is again $Z(p)=1$ and $\widetilde{\Gamma}_{5 a}^{\mu}(p, p+q)=\lambda_{a} \gamma^{\mu} \gamma_{5}$. This may not be a bad approximation if the actual momentum dependence of $Z(p)$ and $\tilde{\Gamma}_{5 a}^{\mu}(p, p+q)$ can be neglected over the momentum range relevant to our final results.

We may consider the WT identity in our model analogous to the one in (10). At tree order it reads 
$-\mathrm{i} q_{\mu} \Gamma_{5 a}^{\mu}(p, p+q)=S^{-1}(p+q) \lambda_{a} \gamma_{5}+\lambda_{a} \gamma_{5} S^{-1}(p)+\mathrm{i} f_{\pi} G_{\pi a}(p, p+q)$.

The right-hand side reduces to $-\mathrm{i} q_{\mu} \lambda_{a} \gamma^{\mu_{\gamma}} \gamma_{5}$ as expected.

We now proceed to derive the effective chiral lagrangian. The transformation properties of $\pi(x)$ and chiral symmetry will ensure that $\mathscr{L}_{\text {eff }}(\pi)$ is a function of $U(x) \equiv \exp \left[-2 \mathrm{i} \pi(x) / f_{\pi}\right]$. We expand $-\operatorname{tr} \log S_{\pi}^{-1}$ in powers of $\pi$ by writing

$-\operatorname{tr} \log S_{\pi}^{-1}=-\operatorname{tr} \log S^{-1}-\operatorname{tr} \log [\delta(x-y)+S \delta \Sigma(x, y)]$,

where $S^{-}(x-y) \equiv \phi_{x} \delta(x-y)+\Sigma(x-y)$ and $\delta \Sigma(x, y)=\Sigma_{\pi}(x, y)-\Sigma(x-y)$. Expanding the log gives a quark loop with any number of insertions of $\delta \Sigma$, the multi-GB vertex function. This may be transformed to momentum space and expanded in powers of the momenta associated with the various external $\pi$ fields. We may integrate over the loop momentum and then convert the result to a local lagrangian density with terms having arbitrary numbers of derivatives and GB fields. We have in the end a derivative expansion of the original nonlocal theory.

We first check that no GB mass appears in $\mathscr{L}_{\text {eff }}(\pi)$. The relevant diagrams are in fig. (1) and their sum vanishes at $p^{2}=0$. We next require the standard normalization for the kinetic term, $\frac{1}{2} \partial^{\mu} \pi_{a}(x) \partial_{\mu} \pi_{a}(x)$. We extract the $p^{2}$ piece from the diagrams in fig. 1 and denote the coefficient of $\frac{1}{2} p^{2} \pi_{a} \pi_{a}$ as $F\left(p^{2}\right) . F\left(p^{2}\right)$ is proportional to $f_{\pi}^{-2}$ and so the constraint $F(0)=1$ yields a formula for $f_{\pi}\left[\Sigma^{\prime}(q) \equiv \mathrm{d} \Sigma(q) / \mathrm{d} q^{2}\right]$,

$f_{\pi}^{2}=\frac{N_{\mathrm{c}}}{(2 \pi)^{2}} \int \mathrm{d} q^{2} q^{2} \Sigma(q)\left(\frac{\Sigma(q)-\frac{1}{2} q^{2} \Sigma^{\prime}(q)}{\left[q^{2}+\Sigma(q)^{2}\right]^{2}}-\frac{C \Sigma^{\prime}(q)+\frac{1}{2} q^{2} \Sigma^{\prime \prime}(q)}{q^{2}+\Sigma(q)^{2}}\right)$.

This integral converges for any reasonable $\Sigma(p)$, as would emerge from a gap equation in a gauge theory. $C$ is an arbitrary constant which depends on the coefficients in (6). For example, when $a=1, b, c, \ldots=0$ and $T=1$, then $C=1$. Unless $C$ vanishes, our result differs from the Pagels-Stokar (PS) result [3].

To show where the discrepancy arises we note that PS determine $f_{\pi}$ from the amplitude for annihilation of a GB by an axial current. For this they use the diagram in fig. 2 where they use eq. ( 9 ) for the GB vertex and $\lambda_{a} \gamma^{\mu} \gamma_{5}$ for the axial current vertex. But our model allows us to identify additional GB dependent contributions to the axial current. This may be seen in the derivation of the appropriate axial WT identity. Under a local chiral transformation the change in the lagrangian consists of the usual divergence of the local axial current as well as a nonlocal, GB dependent, $\gamma^{\mu}$ independent contribution. The result is an identity which is pictured to lowest loop order in fig. 3.

This identity relates the two different methods for calculating $f_{\pi}$. The right hand side is $\mathrm{i} f_{\pi}$ times the inverse GB propagator as obtained from the diagrams in fig. 1. The left hand side is the amplitude for the divergence of the axial current to annihilate a GB. The vertex in the second term is due to the additional contribution to the axial current. This term gives the additional term in our result for $f_{\pi}$. That is, the PS method may be used in our model to calculate $f_{\pi}$ only if this contribution is taken into account.

Is there a choice of parameters in our model for which the extra contribution to the axial current vanishes? We find that the answer is no, although it is possible to choose the parameters such that the extra diagram contributing to $f_{\pi}$ vanishes. It is only then that $C=0$ is recovered.

We have already noted that our model incorporates the same quark propagator and the same single GB vertex function as found in the PS analysis. Even so, we have found that chiral symmetry has not been sufficient to
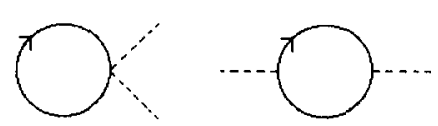

Fig. 1. Two GB contribution to $\mathscr{L}_{\text {eff }}(\pi(x))$.

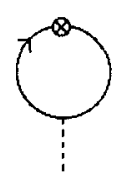

Fig. 2. Pagels-Stokar contribution to $f_{\pi}$. 


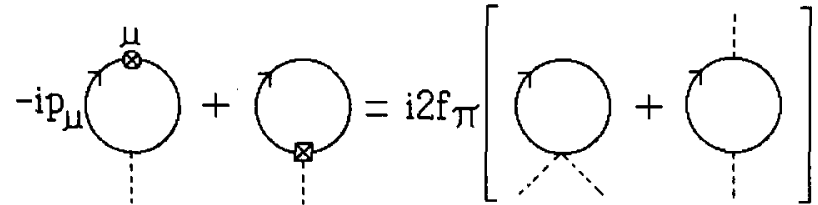

Fig. 3. Ward-Takahashi identity relating two methods for calculating $f_{\pi}$.

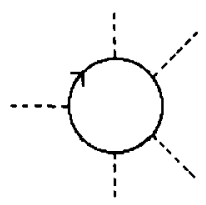

Fig. 4. Five GB contribution to the Wess-Zumino term.

uniquely determine $f_{\pi}$. We have traced the apparent uniqueness of the PS result to the omission of a certain vertex which should, by chiral symmetry, be present in lowest order dynamical perturbation theory.

We next consider the Wess-Zumino term. (Here we assume that there are more than two flavors of quarks). For example we find that the five GB piece of the Wess-Zumino term appears in the five GB term in the derivative expansion. It only receives a contribution from the diagram in fig. 4 and thus it does not depend on the multi-GB couplings. We find the following:

$\mathscr{L}_{\mathrm{WZ}}=\frac{8 N_{\mathrm{c}}}{5 \pi^{2} f_{\pi}^{5}} \operatorname{Tr}\left(\lambda_{a} \lambda_{b} \lambda_{c} \lambda_{d} \lambda_{e}\right) \epsilon^{\mu \nu \tau \kappa}\left(\partial_{\mu} \pi_{a}\right)\left(\partial_{\nu} \pi_{b}\right)\left(\partial_{\tau} \pi_{c}\right)\left(\partial_{\kappa} \pi_{d}\right) \pi_{e} \int \mathrm{d} q^{2} q^{2} \Sigma(q)^{4} \frac{\Sigma(q)^{2}-2 q^{2} \Sigma(q) \Sigma^{\prime}(q)}{\left[q^{2}+\Sigma(q)^{2}\right]^{5}}$.

Here the functional derivative with respect to $\Sigma(q)$ turns out to vanish and we find that the value of the integral is $\frac{1}{12}$ for any nonzero $\Sigma(q)$ for which the integral converges. Thus the apparent dependence on $\Sigma(q)$ is illusory and our model generates the correct coefficient of the Wess-Zumino term.

We may also consider higher derivative terms involving $\epsilon^{\mu \nu \tau \kappa}$. Such terms in the chiral lagrangian are constructed from $U(x)$ and are not "anomalous" in any way. We obtain from fig. 4 the following expression, where $F\left(p_{1}, p_{2}, p_{3}, p_{4}\right)$ replaces the integral in (14):

$$
\begin{aligned}
& \epsilon^{\mu \nu \tau \kappa} p_{1 \mu} p_{2 \nu} p_{3 \tau} p_{4 \kappa} F\left(p_{1}, p_{2}, p_{3}, p_{4}\right) \\
& \quad \equiv \frac{1}{2^{5}} \int \mathrm{d} q^{2} q^{2} \epsilon^{\mu \nu \tau \kappa}\left\{q_{\mu} p_{1 \nu} p_{2 \tau} p_{3 \kappa}\left[-\Sigma\left(z_{4}\right)+\Sigma\left(z_{3}\right)\right]+q_{\mu} p_{1 \nu} p_{2 \tau} p_{4 \kappa}\left[\Sigma\left(z_{3}\right)-\Sigma\left(z_{2}\right)\right]\right. \\
& \left.\quad+q_{\mu} p_{1 \nu} p_{3 \tau} p_{4 \kappa}\left[-\Sigma\left(z_{2}\right)+\Sigma\left(z_{1}\right)\right]+q_{\mu} p_{2 \nu} p_{3 \tau} p_{4 \kappa}\left[\Sigma\left(z_{1}\right)-\Sigma(q)\right]+p_{1 \mu} p_{2 \nu} p_{3 \tau} p_{4 \kappa} \Sigma(q)\right\} \\
& \quad \times\left[\Sigma\left(z_{1}\right)+\Sigma(q)\right]\left[\Sigma\left(z_{2}\right)+\Sigma\left(z_{1}\right)\right]\left[\Sigma\left(z_{3}\right)+\Sigma\left(z_{2}\right)\right]\left[\Sigma\left(z_{4}\right)+\Sigma\left(z_{3}\right)\right]\left[\Sigma(q)+\Sigma\left(z_{4}\right)\right] \\
& \quad \times\left[\Delta(q) \Delta\left(z_{1}\right) \Delta\left(z_{2}\right) \Delta\left(z_{3}\right) \Delta\left(z_{4}\right)\right]^{-1}
\end{aligned}
$$

where $z_{1} \equiv q-p_{1}, z_{2} \equiv q-p_{1}-p_{2}, z_{3} \equiv q-p_{1}-p_{2}-p_{3}, z_{4} \equiv q-p_{1}-p_{2}-p_{3}-p_{4}$, and $\Delta\left(q^{2}\right) \equiv q^{2}+\Sigma(q)^{2}$. This determines the five GB amplitude involving $\epsilon^{\mu \nu \tau \kappa}$ to all orders in momenta.

We now turn to other four derivative terms in the chiral lagrangian. The four derivative four GB terms are determined by the diagrams in fig. 5 . When all four momenta are nonzero these diagrams contribute to terms of the form
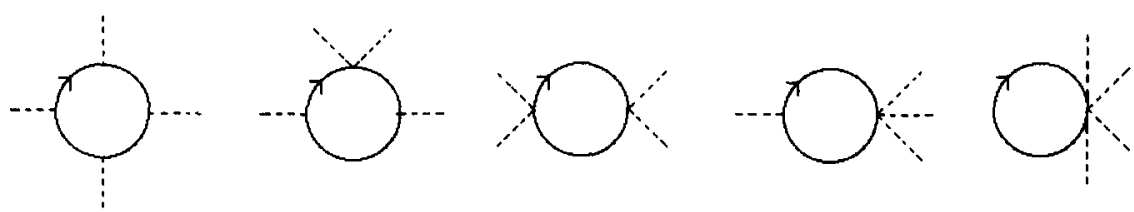

Fig. 5. Four GB contribution to $\mathscr{L}_{\text {eff }}(\pi(x))$. 
$\left[\beta_{1} \operatorname{Tr}\left(\partial^{\mu} \pi \partial_{\mu} \pi \partial^{\nu} \pi \partial_{\nu} \pi\right)+\beta_{2} \operatorname{Tr}\left(\partial^{\mu} \pi \partial^{\nu} \pi \partial_{\mu} \pi \partial_{\nu} \pi\right)\right] / f_{\pi}^{4}$

We would like to give some idea of how $\beta_{1}$ and $\beta_{2}$ may change when one goes from the case $\Sigma(p)=m$, a constant, to a more realistic $\Sigma(p)$. To do this we calculate $\beta_{1}$ and $\beta_{2}$ for a particular set of coefficients in (6): $a=1, b, c$, $\ldots=0$ and $T=1$. The resulting integral expressions for $\beta_{1}$ and $\beta_{2}$ are too lengthy to be reproduced here.

In the chiral lagrangian there are three independent terms which make contributions to $\beta_{1}$ and $\beta_{2}$ :

$\mathscr{L}_{4 \partial}=B_{1} \operatorname{Tr}\left(\partial^{\mu} U^{\dagger} \partial_{\mu} U \partial^{\nu} U^{\dagger} \partial_{\nu} U\right)+B_{2} \operatorname{Tr}\left(\partial^{\mu} U^{\dagger} \partial^{\nu} U \partial_{\mu} U^{\dagger} \partial_{\nu} U\right)+B_{3} \operatorname{Tr}\left(\partial^{2} U^{\dagger} \partial^{2} U\right)$.

The $B_{3}$ term contains the term $\beta_{3} \operatorname{Tr} \partial^{2} \pi \partial^{2} \pi / f_{\pi}^{2}$. This in turn is determined by the $p^{4}$ piece of the two GB diagrams in fig. 1. With this the $B_{i}$ 's are determined: $B_{1}=\frac{1}{16} \beta_{1}-\frac{1}{4} \beta_{3}, B_{2}=\frac{1}{16} \beta_{2}, B_{3}=\frac{1}{4} \beta_{3}$. In Minkowski space all the four derivative coefficients pick up an extra minus sign.

In the $\Sigma(p)=m$ case we obtain $\beta_{1 m}=0, \beta_{2 m}=-N_{\mathrm{c}} / 12 \pi^{2}, \beta_{3 m}=-N_{\mathrm{c}} / 24 \pi^{2}$. In the case of SU(2) $\times \mathrm{SU}(2)$ we may write the $\beta_{1}$ and $\beta_{2}$ terms in (16) as

$\left[\alpha_{1} \partial^{\mu} \pi_{a} \partial_{\mu} \pi_{a} \partial^{\nu} \pi_{b} \partial_{\nu} \pi_{b}+\alpha_{2} \partial^{\mu} \pi_{a} \partial^{\nu} \pi_{a} \partial_{\mu} \pi_{b} \partial_{\nu} \pi_{b}\right] / f_{\pi}^{4}$,

where $\alpha_{1}=\frac{1}{8}\left(\beta_{1}-\beta_{2}\right)$ and $\alpha_{2}=\frac{1}{4} \beta_{2}$. Then these values for $\beta_{1 m}$ and $\beta^{2 m}$ may be seen to agree with, for example, eq. (2.4) (with $\square \pi=0$ ) of ref. [4]. Similarly the $\beta_{3 m}$ result gives $\left(-N_{\mathrm{c}} / 48 \pi^{2} f_{\pi}^{4}\right) \partial^{2} \pi_{a} \partial^{2} \pi_{a}$; this also agrees.

Also in the SU (2) $\times \mathrm{SU}(2)$ case we may quote values [5] extracted from experiment; renormalized at 0.5 $\mathrm{GeV}$ they read $\alpha_{1}^{\mathrm{expt}}=-0.010, \alpha_{2}^{\mathrm{expt}}=0.0075$. This may be compared to $\alpha_{1}=-0.0032, \alpha_{2}=0.0063$ for the case $\Sigma(p)=m$.

A more realistic choice for $\Sigma(p)$ is motivated by properties of solutions to a gauge theory gap equation. Quite generally, $\Sigma(p)$ is a monotonically decreasing function of $p$ which is finite at $p=0$ and which for large $p$ falls like $1 / p^{2}$ times a power of $\log p^{2}$. We may neglect the $\log$ since our expressions are quite insensitive to the large $p$ behavior. We take $\Sigma(p)=\delta m^{3} /\left(m^{2}+\gamma p^{2}\right)$ for three different values of $\gamma:\left(\frac{1}{2}, 1,2\right)$. A normalization condition $\Sigma(m)=m$ fixes $\delta$. [We note that the $\beta_{i}$ 's are invariant under the scaling $\Sigma(p) \Rightarrow \sigma \Sigma(p / \sigma)$ ]. We obtain $\alpha_{1}=(-0.0037,-0.0044,-0.0055)$ and $\alpha_{2}=(0.0069,0.0074,0.0081)$. We also obtain $f_{\pi} / m=(0.30,0.28$, 0.27 ) from (13) $(C=1)$ to be compared with the Pagels-Stokar formula $(C=0)$ which gives $f_{\pi} / m=(0.27$, $0.25,0.23)$. $m$, crudely speaking, is the constituent mass.

Thus far we have assumed a vanishing current quark mass. A current quark mass may be introduced by adding a GB independent mass term to $S_{\pi}^{-1}$ :

$S_{\pi}^{-1}(x, y) \equiv \partial_{x} \delta(x-y)+\Sigma_{\pi}(x, y)+m_{\mathrm{q}} \delta(x-y)$.

For example the two diagrams in fig. 1 now yield a mass term for the GB and to lowest order in $m_{\mathrm{q}}$ we obtain the standard result

$m_{\pi}^{2}=-2 m_{\mathrm{q}}\langle\bar{\psi} \psi\rangle / f_{\pi}^{2}$,

where $\langle\bar{\psi} \psi\rangle=\left[-4 N_{\mathrm{c}} /(2 \pi)^{4}\right] \int \mathrm{d}^{4} q \Sigma(q) /\left[q^{2}+\Sigma(q)^{2}\right]$. We may also introduce gauge fields. The nonlocality makes the gauging of our model nontrivial, but we have been able to reproduce for example the Wess-Zumino terms with gauge fields. Further implications of current quark masses and gauge fields in our model will be considered elsewhere.

In summary we have considered a nonlocal generalization of the nonlinear sigma model in which GBs appear as fluctuations in the order parameter $\Sigma(p)$. This better represents generic features of chiral symmetry breaking in gauge theories. But we find that chiral symmetry alone is insufficient to completely determine the multi-GB couplings. And thus the low energy chiral lagrangian has some undetermined dependence on derivatives of $\Sigma(p)$.

Is there some natural way to constrain our model further? We return to the local sigma model with only the minimal, nonderivative GB-quark couplings in (1). That model has the axial current vertex function equal to $\lambda_{a} \gamma^{\mu} \gamma_{5}$ and it has no tree order vertex functions with multiple axial currents and two quark fields. In our model 
we have the same axial current vertex function but we find that it is impossible to arrange for all tree order multiple axial current vertex functions to vanish. This may be traced to the nonabelian nature of the chiral symmetry. In the special case of one flavor we may construct a version of our model with $U(1)_{L} \times U(1)_{R}$ chiral symmetry. Here it is possible to make all multiple axial current vertex functions vanish. And this constraint turns out to specify $\Sigma_{\pi}(x, y)$ uniquely. It yields $C=0$ in the formula for $f_{\pi}$ since in this case the pion dependent term in the axial current vanishes, unlike the nonabelian case. In the nonabelian case the best we can do is to require the vanishing of vertex functions in which all axial currents have commuting generators. This also determines $\Sigma_{\pi}(x, y)$; implications (which include $C=0$ ) will be considered elsewhere.

We thank B. Bardeen, M. Sutherland, and E. Swanson for useful discussions. This research was supported in part by the Natural Sciences and Engineering Research Council of Canada.

\section{References}

[1] I.J.R. Aitchison and C.M. Fraser, Phys. Lett. B 146 (1984) 63; Phys. Rev. D 31 (1985) 2605;

A.A. Andrianov, Phys. Lett. B 157 (1985) 425;

J. Balog, Phys. Lett. B 149 (1984) 197;

L.-H. Chan, Phys. Rev. Lett. 55 (1985) 21;

A. Dhar, R. Shankar and S.R. Wadia, Phys. Rev. D 31 (1985) 3256;

D. Ebert and H. Reinhardt, Phys. Lett. B 173 (1986) 453; Nucl. Phys. B 271 (1986) 188;

R. Mackenzie, F. Wilczek and A. Zee, Phys. Rev. Lett. 53 (1984) 2203;

D.W. McKay and H.J. Munczek, Phys. Rev. D 32 (1985) 266;

R.I. Nepomechie, Ann. Phys. 158 (1984) 67;

P.D. Simic, Phys. Rev. D 34 (1986) 1903;

M. Volkov, Ann. Phys. 157 (1984) 282;

A. Zaks, Nucl. Phys. B 260 (1985) 241;

J.A. Zuk, Z. Phys. C 29 (1985) 303.

[2] D.W. McKay, H.J. Munczek and B.-L. Young, Phys. Rev. D 37 (1988) 195;

D.W. McKay and H.J. Munczek, Phys. Rev. D 39 (1989) 888;

A. Barducci, R. Casalbuoni, S. De Curtis, D. Dominici and R. Gatto, Phys. Rev. D 38 (1988) 238.

[3] H. Pagels and S. Stokar, Phys. Rev. D 20 (1979) 2947;

R. Jackiw and K. Johnson, Phys. Rev. D 8 (1973) 2386.

[4] I.J.R. Aitchison, C.M. Fraser and P.J. Miron, Phys. Rev. D 33 (1986) 1994.

[5] J. Donoghue, C. Ramirez and G. Valencia, Phys. Rev. D 38 (1988) 2195. 